\title{
Research Progress of Concrete Healing Materials and Technologies in Marine Engineering
}

\author{
Yanxuan Ma ${ }^{1, a}$, Xin Lei, b, Yingrui Zhang ${ }^{1, c}$, Jinhua Wang ${ }^{1, d}$ \\ ${ }^{1}$ School of Civil Engineering, Qingdao University of Technology, Qingdao, China \\ ayxma@qut.edu.cn, b1207183066@qq.com, '870529799@qq.com, d1614377950@qq.com
}

Keywords: Marine Concrete, Crack, Healing Materials, Healing Technology, Corrosion Prevention Abstract. Because of the different environment and existing conditions, the damages of structural concrete are different. The typical problems of ocean engineering concrete corrosion, crack, and durability are discussed. The healing materials used in marine engineering, the current development situation of relative technology, and the method to enhance the concrete durability have been summarized. The existing problems in this field has been put forward and the expected trend for concrete healing development has been discussed.

\section{Introduction}

As concrete has the characteristic of high strength and low cost, it is more and more frequently used in Marine engineering. Under the condition of varying temperature, humidity and variable load, cracks occur in marine concrete during the service period. Concrete breaks under worse situation. Besides, being positioned in the salty environment with more ions and influenced by the physical or chemical factors, the rebar of concrete may get rusty, causing the decline in mechanical property or even causing safety accidents. Therefore, the healing material and healing technology of marine concrete have become a hot topic and great attention has been paid by scientific workers. Due to unique serving environment of protection coatings, the traditional methods to healing materials cannot stand the erosion for a long time.

\section{Current situation of marine concrete}

As a widely used engineering material, the study of concrete is a hot topic in current and future research. Buddha invented the reinforced concrete and concrete technologies are developed rapidly after World War II. 1.06 million tons of concrete are used in Great Belt Project [1].King Fahd bridge, domestic bridge of hangzhou bay bridge, the Yangtze river delta bridge are expected to last 100 years. In fact, the life span of marine engineering concrete is very short, generally only thirty years or so. It is hard to be used in projects that are supposed to last one hundred years. In addition, the maintainance cost is also high. China spends at least 1.2 trillion yuan in concrete maintainance in 2010 [2]. The domestic and overseas scholars established the corresponding models to evaluate the service life of concrete. Yuan[3] establishes the model by the diffusion characteristics of chloridion, which is used to indicate concrete structure damage conditions so that its life span can be predicted.

\section{Marine concrete damage forms and mechanism}

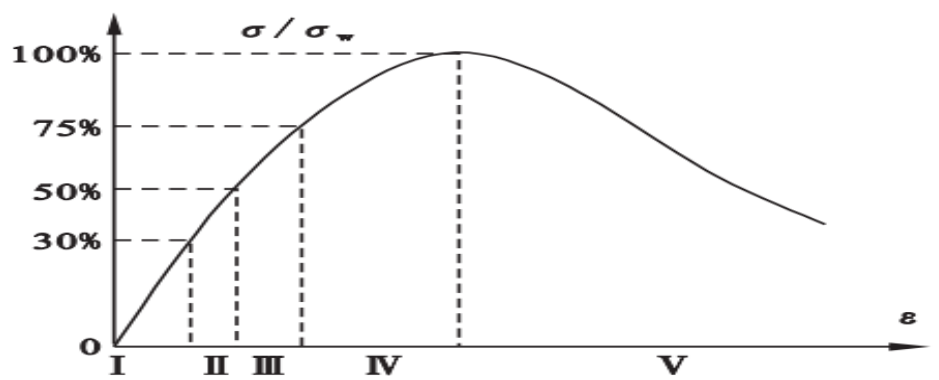

Fig.1 Concrete crack evolution stage[4] 
Fig. 1 indicates the change of the concrete crack. Concrete has high compressive strength and low tensile strength. Under the influence of load and being vulnerable to tensile stress, cracks occur, thus causing the instability of concrete. Lots of the hydration heat come into being when hardened, which causes temperature difference with the outside world, and in turn the temperature stress shows. It breaks with over temperature stress. With the greeze-thaw damage, stress is emerged when concrete surface freezes. Low osmotic pressure can cause water pressure. With higher pressure, concrete cracks. Hasan [5] thought concrete freeze-thaw damage was caused by fatigue damage. Alkali environment of concrete is damaged due to the watering of carbon dioxide and carbonation, which accelerates the corrosion speed of bar.

\section{Current healing technologies}

Crack controlling and restoration are the keys to restore the water proofing property and durability in marine engineering. Traditionally, the healing technologies are surface healing method, local healing method and grouting method. There are certain disadvantages for these methods. Therefore, the new healing technology has been extensively studied. Currently, the domestic and overseas technologies include: microbial action, crystal penetration technology, built-in viscose fluid tube method, crystal precipitation, microcapsule self-healing method, and carbon fiber reinforced method.

\section{Microbial action}

In this method, soil bacteria is used to produce calcium carbonate deposits. The principle is that urea is disintegrates into ammonia and carbon dioxide by urea ferment. The release of ammonia can increase the $\mathrm{pH}$ of local environment, accumulating $\mathrm{CaCO}_{3}$ through physical and chemical reaction. Qian [6] obtains deposition by the reaction between carbon dioxide and calcium compound resulting from the breathing of aerobic bacteria in the soil. This method is ecological and has a good healing effect. But the high alkali environment of the concrete can cause the massive death of bacteria. Due to the limited life of bacteria, the long-term concrete healing cannot be guaranteed.

\section{Crystal penetration technology}

The active chemical substances can catalyze a series of reactions of the un-hydrated granule through penetration, which can generate insoluble crystals. With gels, the crystal can block the cracks. When the cracks occur and water penetrates into the concrete, the chemical substances can catalyze the process of crystallization, which generate crystals to block the cracks. It has a bright future to healing the concrete with this hydration products. This method needs the chemical reaction of water and can realize the self-reparation of concrete, but this can be only used to healing the small cracks.

The built-in viscose fluid tube method

Carolyn [7] buries a hollow glass fiber, injected with acetal polymer solution, into the concrete. When the concrete cracks, the solution flows and restores the crack. In1996, the ATR Lab of Illinois in the United States healings deck crack by embedding a healing pipe which consists concrete healing adhesive in the concrete. The method can heal cracked part partially and thus can enhance the flexural performance of concrete. But it is difficult to control the number of fibers and such excess or deficiency cannot have a very good healing effect.

\section{Microcapsule self-healing}

The key to the method is to have a "target" healing to the cracks. The mechanism is illustrated in fig.2. When the concrete cracks, the microcapsule breaks under the stimulus, and healing agent flows to heal cracks. The types can be one-wall and double-wall healing. It is the special applications on ocean engineering protection coatings that it will become an important research field in the future. Due to the poor match between the coating and microcapsule, a new type microcapsule needs to be 
designed so that the reasonable parameters can be gotten and the durability of solution needs to be improved.

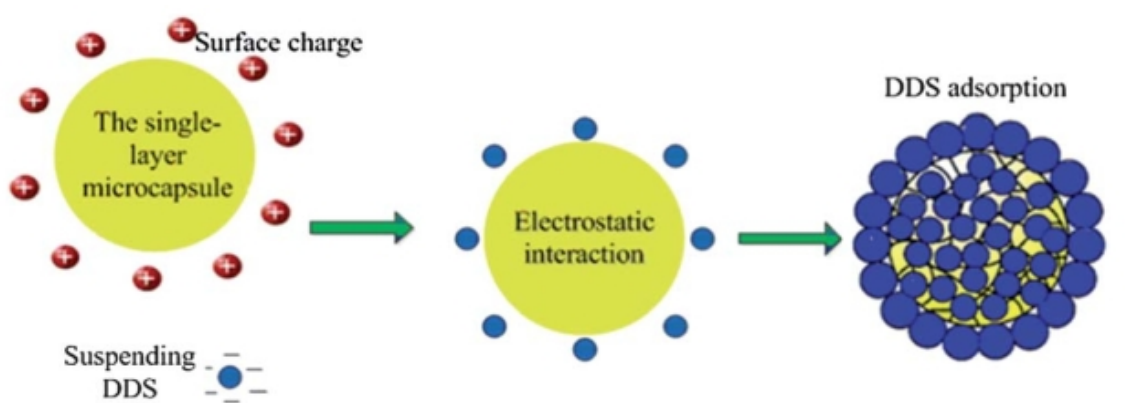

Fig.2 Adsorption mechanism of the DDS/microcapsules[8]

\section{CFRP-strengthened reinforced concrete}

As a newly-developed concrete structure process technology, this method was adopted in Japan in 1980s. Later, it has been used in Switzerland and England successively. During the recent decades, this method has been used to reinforce the bridge in China. By adding the fiber reinforced plastics into the structure of concrete, the concrete can function normally even under pressure. Thus, this newly-developed material has been used to enhance the mechanical property of concrete. This method can heal the concrete in a much better way and it is good in chemical performance and feasibility. But because of high cost and lacking accurate statistics to show the mechanical property of concrete, it is difficult to use this method to heal the concrete with specific shapes.

\section{Current healing materials}

\section{Healing polymer materials}

Polyurea coating: Polyurea coatings is a kind of elastomer, made by the reaction of isocyanate and amino. It has the advantages of high strength, high elasticity, and fast curing speed. But the coating is not sensitive to water, its permeability is not good and it is not easy to be attached to the concrete surface. Epoxy resin coating [9]: epoxy resin coating is the most widely used solvent heavy-duty coating at present. Although its mechanical strength is big and performance is excellent, it is poorly soluble in water and bad for ecosystem. Thomas shows it can adapt to strong alkaline seawater environment. Chonkaew modified epoxy resin coating by the combination of end carboxyl nitrile rubber and organic clay. Grasbock develops an anti-corrosion primer paint with high performance which has excellent anti-corrosion performance.

Healing inorganic materials

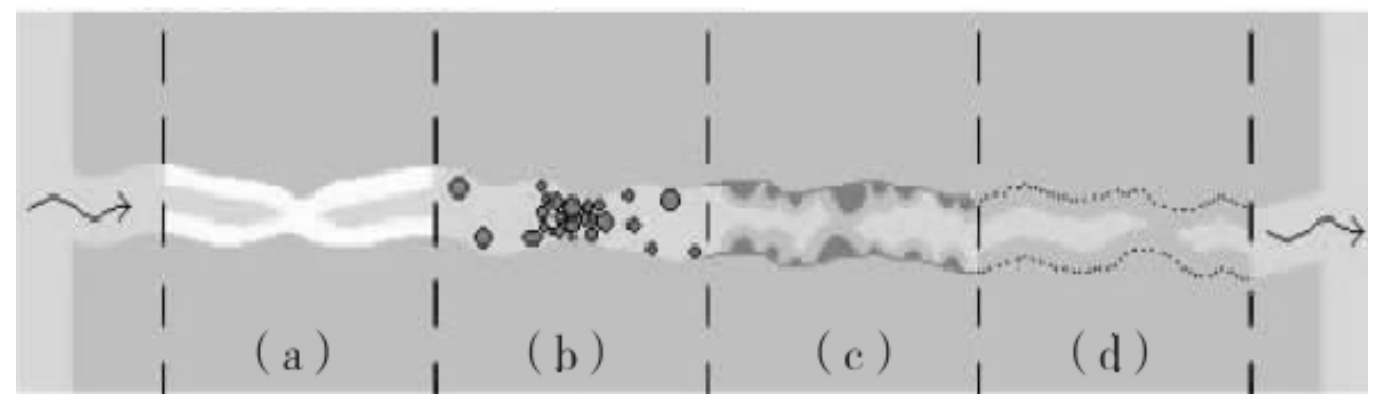

Fig.3 Self-healing way to cement-based materials[10]

Cement-based materials refer to engineering materials with cement as the gel material, which are divided into cement matrix material, binder resin matrix material, and cement-based materials. Cement matrix materials have high strength, which are often used in concrete engineering. The self-healing way of cement-based materials is illustrated in fig.3, but the curing period for this material is too long. 
Silicon gel and polyurethane are also used as microbial carrier to healing cement-based materials, which improves the mechanical properties of concrete. When concrete cracks, water is used as the carrier, and active substances dormancy are broken to generate a new crystal to block the crack.

\section{Conclusions}

The traditional concrete healing targets at the macroscopic visible cracks, which is hard to solve fundamental problems and to control crack regeneration. Although more and more new types of marine engineering concrete healing materials and technologies have been developed, there is a long distance to use them in a large scale. New polymer materials and Nano-materials will be hot topics because of their light and excellent performance. Optimizing the traditional healing technologies with appropriate additives and researching on new composite materials are also developing. The study of marine engineering concrete healing will be focused on the aspects of less toxicity, more intelligent, and more ecological.

\section{Acknowledgements}

This work was financially supported by the National Natural Science Foundation of China (51408330) and the Foundation for Outstanding Young Scientist in Shandong Province (BS2014CL031).

\section{References}

[1] Ma SD, Zhao J. Marine fouling organisms to marine concrete engineering corrosion analysis. Journal of Guangxi Academy of Sciences, 2015, 31(3): 209-213.

[2] Hou BR, Li WH, Jin ZQ, et al. Technology of marine concrete corrosion and healing reinforcement[M]. Beijing: Science Press, 2012: 1-3.

[3] Yuan CF, Niu DT. Life prediction of the concrete structure based on the reliability theory[A]. Journal of Wuhan university of Technology, 2013, 35(4): 73-79.

[4] Zhi S, Fang LM, Jing G. Review of studies on mechanical property and micro mechanism of concrete [J]. Concrete, 2015, 309(7): 11-17.

[5] Hasan M, Ueda T, Sato Y. Stress-strain relationship of frost damaged concrete subjected to fatigue loading[J]. Journal of Materials in Civil Engineering, 2010, 20(1): 37-45.

[6] Qian CX, Mian L, Pan QF. Microbial mineralization in self-healing concrete calcite formation mechanism[J]. Journal of silicate, 2013, 4(5): 620-626.

[7] Carolyn D. Healing and prevention of damage due to transverse shrinkage cracks in bridge decks. Proceedings of SPIE-The International Society for Optical Engineering. 1999, 3671: 253-256.

[8] Tian R, Fu X, Zheng Y, et al. The preparation and characterization of double-layer microcapsules used for the self-healing of resin matrix composites[J]. Journal of Materials Chemistry, 2012, 22(48): 25437-25446.

[9] Ping KL, Sun CT, DT Niu. Marine environmental anticorrosive coatings research and development[J]. Concrete, 2013, (4): 52-55.

[10] Heide NT. Crack Healing in Hydrating Concrete[D]. Netherlands: Delft University of Technology, 2005: 4-10. 